\title{
Educação em saúde no cuidado de pacientes oncológicos com o uso da auriculoterapia no manejo da dor
}

\author{
Health education in the care of oncological patients with the use of auriculotherapy in \\ pain management
}

\section{Educación en salud en la atención del paciente oncológico con el uso de auriculoterapia en el tratamiento del dolor}

Patrick Roberto Gomes Abdoral ${ }^{1 *}$, Adriana Esther Vicente Freire ${ }^{2}$, Gabriel Figueiredo Santos ${ }^{2}$, Larissa Siqueira Rodrigues ${ }^{2}$, Felix Ferreira Furtado ${ }^{2}$, Eduarda de Melo Rodrigues e Oliveira ${ }^{2}$, Paula Gabriela Rezek ${ }^{3}$, Mayra Herminia Simões Hamad Farias do Couto², Susanne Cristine Brito e Silva², Cláudia Jeane Claudino de Pontes Miranda².

\section{RESUMO}

Objetivo: Verificar o efeito da auriculoterapia no manejo da dor de pacientes oncológicos em tratamento quimioterápico. Métodos: Foram avaliados 12 pacientes no estadiamento II, III ou IV, submetidos a tratamento quimioterápico que apresentavam queixa álgica a partir da EVA-3. Foi coletado informações sobre o estado geral, caso o paciente se encaixasse nos critérios pré-estabelecidos o mesmo era convidado a participar do estudo. Para analisar o efeito da auriculoterapia no manejo da dor, utilizou-se o Teste T pareado para as variáveis referentes (EVA) antes e após as sessões. Resultados: Após a análise verificou-se diferença estatisticamente significativa na diminuição da intensidade da dor e consumo de analgésico por parte dos participantes avaliados. Quando perguntados sobre o uso de analgésicos antes das sessões, $41,67 \%$ relataram fazer uso. Após as sessões, quando feita a mesma pergunta, $16,67 \%$ relataram fazer uso. Conclusão: Portanto, a auriculoterapia é uma terapia complementar em saúde, promissora para o manejo da dor e redução do consumo de analgésicos por pacientes oncológicos, entretanto, novos estudos baseados em ensaios clínicos randomizados e controlados com amostras maiores e homogêneas devem ser realizados afim de evidenciar o aprimoramento dessa prática terapêutica.

Palavras-chave: Educação em saúde, Auriculoterapia, Dor oncológica, Terapias complementares, Medicina integrativa.

\begin{abstract}
Objective: To verify the effect of auriculotherapy in the treatment of cancer patients undergoing chemotherapy. Methods: Twelve patients at stage II, III or IV were treated, undergoing chemotherapy and presenting pain complaints from VAS-3. Information on general status was collected, if the patient met the pre-included criteria, he was invited to participate in the study. To analyze the effect of auriculotherapy on pain management, use the paired t-test for (VAS) variables before and after completion. Results: After the analysis, a statistically decreased difference in pain intensity and analgesic consumption by registered participants was verified. When asked about the use of analgesics before classes, $41.67 \%$ reported using them. After the choices, when asked the same question, $16.67 \%$ reported using it. Conclusion: Therefore, auriculotherapy is a promising complementary health therapy for pain management and reduction of analgesic consumption by cancer patients, however, new studies based on randomized and controlled clinical trials with larger and homogeneous ones should be carried out in order to evidence the improvement of this therapeutic practice.
\end{abstract}

Keywords: Health education, Auriculotherapy, Oncologic pain, Complementary therapies, Integrative medicine.

${ }^{1}$ Faculdade Cosmopolita, Belém - PA. *E-mail: patrickabdoral@gmail.com

2 Universidade da Amazônia (UNAMA), Belém - PA.

${ }^{3}$ Oncológica do Brasil, Belém - PA.

SUBMETIDO EM: 7/2021 


\section{RESUMEN}

Objetivo: Verificar el efecto de la auriculoterapia en el tratamiento de pacientes oncológicos sometidos a quimioterapia. Métodos: Se trató a doce pacientes en estadio II, III O IV, sometidos a quimioterapia y que presentaban quejas de dolor por EVA-3. Se recogió información sobre el estado general, si el paciente cumplía con los criterios pre-incluidos, se le invitaba a participar en el estudio. Para analizar el efecto de la auriculoterapia en el manejo del dolor, utilice la prueba t pareada para las variables (EVA) antes y después de la finalización. Resultados: Después del análisis, se verificó una diferencia estadísticamente disminuida en la intensidad del dolor y el consumo de analgésicos por parte de los participantes registrados. Cuando se les preguntó sobre el uso de analgésicos antes de las clases, el 41,67\% informó que los usaba. Después de las opciones, cuando se le hizo la misma pregunta, el 16,67\% informó que la usaba. Conclusión: Por tanto, la auriculoterapia es una prometedora terapia complementaria de salud para el manejo del dolor y la reducción del consumo de analgésicos por parte de los pacientes oncológicos, sin embargo, se deben realizar nuevos estudios basados en ensayos clínicos aleatorizados y controlados con otros más amplios y homogéneos para evidenciar la mejora esta práctica terapéutica.

Palabras clave: Educación en salud, Auriculoterapia, Dolor en cáncer, Terapias complementarias, Medicina integral.

\section{INTRODUÇÃO}

O câncer é considerado uma doença de caráter crônico degenerativo, multifatorial, de crescimento anormal de células, prejudicando o funcionamento normal do organismo. Dados presentes na literatura médica mundial apontam que existem centenas de tipos de câncer diferentes (MINISTÉRIO DA SAÚDE, 2019).

Os riscos relacionados ao câncer vêm aumentando no mundo por diversos fatores como a crescente no número de habitantes, desenvolvimento socioeconômico e o aumento da longevidade de vida dos indivíduos, tais fatores expõe a população de maneira mais direta aos fatores de risco que possam desencadear o processo cancerígeno e isso impacta diretamente na incidência e por consequência na morbimortalidade populacional pelo câncer. Há dados estatístico que apontam o crescimento acelerado de casos de câncer, até o ano de 2022 pode surgir aproximadamente 625 mil novos registros (BRAY F, et al., 2018).

O aumento na prevalência de câncer está associado com os níveis socioeconômico da população, sendo câncer de pele não melanoma o mais incidente, seguido pelos cânceres de mama e próstata, cólon e reto, pulmão e estômago (MINISTÉRIO DA SAÚDE, 2019). Apesar dos vários órgãos acometidos, a dor oncológica é um elo comum, sendo manifestada em todos os estágios do processo neoplásico (SANTOS MO, et al., 2018). A dor oncológica é um sintoma multifatorial, caracterizada como uma sensação aguda ou crônica, de variadas intensidades, que está relacionada a invasão tumoral, ao tratamento ou a evolução da própria doença, como a dor da lesão (GARCIA TR, et al., 2018).

No que se refere ao controle da dor oncológica, as equipes de saúde ainda apresentam dificuldade de conhecimento quanto a avaliação e manejo clínico deste sintoma subjetivo (LUIZ MM, et al., 2018). Mesmo após a aprovação do método da escada analgésica pela Organização Mundial da Saúde (OMS) (SAYED D, et al., 2018). Desenvolvida pela OMS ela é graduada em 3 graus para guiar uso sequencial de drogas, no tratamento da dor de câncer. O câncer gera um grande impacto nos pacientes e familiares. Por isso a importância de tratamentos adequados que tornem essa caminhada mais confortável e menos angustiante (QUEIROGA HM, et al., 2017).

Desse modo, os serviços em saúde têm a possibilidade de inserir métodos complementares e seguros na tentativa de possibilitar o melhor manejo álgico (WU X, et al., 2017). Nesse contexto, destaca-se a auriculoterapia, que é uma das vertentes de um grupo de práticas terapêuticas que apresenta como base a Medicina Tradicional Chinesa (MTC) não como uma técnica única, mas como coadjuvante e complementar a outros métodos. Sendo assim, uma prática não medicamentosa (MATOS LC, et al., 2021). A mesma tem se destacado no tratamento e controle da dor. Essa terapia utiliza a estimulação de pontos auriculares, a fim equilibrar o organismo (LI T, et al., 2021). 
O qual desencadeia a liberação de substâncias, promovendo a sensação de bem-estar do paciente. Entretanto, as evidências científicas focadas no efeito da auriculoterapia no manejo da dor oncológica ainda são limitadas, devido ao baixo número de publicações acerca da temática (RUELA LO, et al., 2018).

No contexto das Práticas Integrativas e Complementares (PICS), são condutas terapêuticas que fazem uso de recursos com base nos conhecimentos tradicionais, com foco na prevenção das mais diversas doenças como depressão, hipertensão e para pacientes oncológicos. Tais terapias também podem ser usadas como tratamentos de algumas doenças crônicas e para pacientes em cuidados paliativos (MINISTÉRIO DA SAÚDE, 2021).

A auriculoterapia se destaca como uma terapia complementar em saúde, promissora para o manejo da dor em pacientes oncológicos, alguns autores destacam o processo de educação em saúde como a premissa fundamental, com isso a comunicação em saúde passa a ser democrática e participativa, fazendo com que as PICS possam integrar a cada dia mais o cuidado com pacientes oncológicos. A introdução das práticas complementares como auriculoterapia traz uns grandes benefícios aos pacientes submetidos a tratamento quimioterápicos pois é uma terapia não medicamentosa que pode modular o quadro álgico e diminuir a administração de fármacos analgésicos. Para que as práticas sejam aplicadas corretamente e imprescindível que haja um processo de educação em saúde para os profissionais que atuam na linha de frente do tratamento oncológico (MINISTÉRIO DA SAÚDE, 2021).

O profissional da saúde deve realizar suas condutas baseadas em evidencias clinicas e cientificas, isso gera um grande entrave com as terapias integrativas pois a base delas, normalmente, está fundamentada no conhecimento holístico. Tal fator acaba gerando um estigma negativo sobre os métodos, porém tais técnicas que vem se demostrando eficiente no âmbito clinico, nasce aí a importância de se realizar estudos para quantificar a eficiência do método (VALLIM ETA, et al. 2019).

Dado isso, esse estudo constitui, portanto, em verificar o efeito da auriculoterapia no manejo da dor de pacientes oncológicos em tratamento quimioterápico e verificar se houve alguma mudança no consumo de fármacos analgésicos e se houve dados significativos na modulação dos quadros álgicos durante o uso da terapia.

\section{MÉTODOS}

Trata-se de um estudo de coorte, prospectivo, observacional, de centro único e caráter quanti-qualitativo. O estudo foi realizado em uma instituição privada de referência em tratamento oncológico no estado do Pará.

De acordo com a frequência do diagnóstico local, foram integrados a este estudo pacientes de ambos os sexos, na faixa etária entre 18 e 80 anos, sendo o câncer de mama, gástrico, reto e de próstata, com estadiamento II, III ou IV submetidos a tratamento quimioterápico e que apresentavam queixa álgica a partir da Escala Visual Analógica 3 (EVA-3). Todos os pacientes assinaram o Termo de Consentimento Livre Esclarecido (TCLE), conforme preconiza o comitê de ética em pesquisa. Pacientes com alterações cognitivas, ausência do pavilhão auricular e que não desejassem mais participar da pesquisa, foram excluídos deste estudo.

A realização do projeto ocorreu de acordo com o código de Nuremberg, considerando os aspectos éticos em pesquisa com ser humano, e em concordância com a resolução 466/12 do Conselho Nacional de Saúde Número do parecer: 3.329.258.

A abordagem ocorreu inicialmente com uma avaliação do fisioterapeuta responsável nas suítes ou poltronas, sendo coletadas informações sobre o estado geral dos participantes, após a avaliação era analisado se o mesmo era compatível com os critérios de inclusão, caso fosse apto o paciente era convidado a participar do estudo em questão, e em seguida, os pesquisadores aplicavam o questionário sociodemográfico. O questionário foi elaborado pelos pesquisadores embasados na ficha de avaliação fisioterapêutica da clínica na qual o estudo foi realizado, além de compor 13 perguntas referente a auriculoterapia. Após a aplicação do questionário, era explicado ao paciente como o seria o acompanhamento no presente estudo. 
O acompanhamento era efetuado semanalmente, no dia e no horário do tratamento quimioterápico, sendo a auriculoterapia aplicada pelo fisioterapeuta antes, durante ou depois da terapia infusional, sob acompanhamento dos pesquisadores. A técnica era aplicada mediante a queixa que o paciente relatava, no caso da dor era colocado de acordo com o segmento queixoso. Tanto a avaliação quanto a reavaliação foram efetivadas pelos pesquisadores do estudo.

Utilizou-se os pontos auriculares como Shenmen, rim, simpático, ponto analgesia e o ponto correspondente ao local da queixa álgica, de acordo com o mapa auricular. Quando o terapeuta realiza a estimulação do ponto Shenmen são desencadeados efeitos calmantes para o sistema cardiovascular e psicológico, gera modulação de quadros álgicos através da liberação de endorfinas, esse processo ocorre graças ao estimulo gerado no tronco, que é recebida e decodificada na substância cinzenta, local do processamento neural mais sofisticado e distinto, isso gera um processo de modulação que condiciona atividades reflexas nos pontos aplicados posteriormente, isso impede que ocorram desequilíbrios na homeostase corporal do paciente e gerando um processo de prevenção (RUELA LO, et al., 2018).

O ponto auricular do rim pode estimular a eliminação de urolitíases e liberação de toxinas auxiliando no processamento do sangue é realizado pelo nefro, isso promove uma melhora importante no sistema circulatório, melhora a o funcionamento das glândulas endócrinas e melhora 0 as funções do sistema respiratório (PEREIRA FEM e MOREIRA DVQ, 2017).

Quando estimulado o ponto auricular Sistema Neuro Vegetativo (Simpático) ocorre um processo de regulação das funções simpática e parassimpática proporcionando uma homeostase do sistema nervoso autônomo, estimula a suprarrenal na produção de cortisol, o que promove o equilíbrio geral do organismo. Esse ponto possui uma importante ação antiansiolítica, analgésica, anti-inflamatória e de relaxamento de fibras musculares.

O Ponto de analgesia é indicado para dores que acometem o sistema musculoesquelético com origem entre outras, de alterações óssea, articular, ligamentar, traumática, pós-operatória e tensional (ARTIOLI DP, et al., 2019). Além de ser informado sobre a durabilidade da auriculoterapia, por no máximo 5 dias, o mesmo era orientado sobre a estimulação do ponto aplicado, realizando 2 a 3 vezes durante o dia cada ponto. Caso houvesse persistência da sintomatologia referida na avaliação inicial durante a visita de acompanhamento, era reaplicado os pontos em questão e houve uma média de duas seções de auriculoterapia por paciente.

Os materiais que foram utilizados na execução da auriculoterapia eram de uso próprio da clínica, sendo eles apalpador de pressão com mola, placa para semente, ambos da marca DUX, semente de mostarda, pinça, esparadrapo ou micropore da marca Nexcare, álcool 70\% para higienização e um frasco de plástico para armazenar as sementes.

Os dados foram armazenados em planilhas do programa Microsoft Excel 2016 e a análise feita no programa Stata 12.0. As variáveis qualitativas foram descritas por frequência absoluta e relativa, enquanto as quantitativas que apresentaram distribuição normal (Shapiro-Wilk, $p>0,05$ ) foram apresentadas por média $e$ desvio padrão.

Para analisar o efeito da auriculoterapia no manejo da dor, utilizou-se o Teste T pareado para as variáveis referentes à EVA a mesma foi aplicada antes da auriculoterapia ser efetuada e após alguns dias foi efetivada a reavaliação, a fim de determinar se houve diferença estatisticamente significativa entre as médias de dor pré e pós-auriculoterapia. Considerando valor de $p<0,05$.

\section{RESULTADOS}

A população do estudo contou com o número de 14 participantes inicialmente que realizavam tratamento quimioterápico na em um hospital de referência em tratamento de câncer no estado do Pará. As abordagens foram realizadas entre julho e setembro de 2019 no setor ambulatorial da clínica. No decorrer do estudo houve perda amostral, o que levou a uma amostra final de 12 participantes no total.

Os participantes foram caracterizados de acordo com as variáveis sociodemográficas e clínicas (Tabela 1) e quanto ao uso de analgésico no decorrer do tratamento (Tabela 2), sendo a variáveis sobre uso, dosagem, frequência e percepção dolorosa pós auriculoterapia. 
Tabela 1 - Caraterísticas gerais e clínicas dos pacientes oncológicos submetidos a auriculoterapia em uma clínica particular no estado do Pará, 2019, n=12 participantes.

\begin{tabular}{lcc}
\hline CARACTERÍSTICA & N & $\%$ \\
\hline Sexo & & 33,3 \\
$\quad$ Masculino & 8 & 66,7 \\
\hline Feminino & & \\
\hline Diagnóstico & 7 & 58,3 \\
$\quad$ Câncer de mama & 3 & 25,00 \\
Câncer de próstata & 2 & 16,7 \\
$\quad$ Câncer de reto & & \\
\hline Comorbidade & 3 & 25,0 \\
\hline Hipertensão arterial & 2 & 16,7 \\
$\quad$ Diabetes mellitus e Hipertensão arterial & 6 & 50,0 \\
$\quad$ Sem comorbidades & 1 & 8,3 \\
$\quad$ Outros & & 100,00 \\
\hline Tratamento & 12 & \\
\hline$\quad$ Quimioterapia & 62,3 & \\
\hline Idade média & & \\
\hline
\end{tabular}

Legenda: $\mathrm{N}=12$ participantes.

Fonte: Abdoral PRG, et al., 2021.

Observou-se que a maioria dos participantes era do sexo feminino (66,7\%), sendo a média de idade 62,3 anos. O diagnóstico prévio mais prevalente foi de câncer de mama $(58,3 \%)$ e independente do tipo de neoplasia, sendo que metade destes não apresentavam comorbidades além do câncer.

Tabela 2 - Uso de analgésicos e percepção de dor pelos pacientes oncológicos submetidos à auriculoterapia em uma clínica particular no estado do Pará, 2019.

\begin{tabular}{lcccc}
\hline \multicolumn{1}{c}{ PERGUNTA } & \multicolumn{2}{c}{ SIM } & \multicolumn{2}{c}{ NÃO } \\
\cline { 2 - 5 } & $\mathbf{N}$ & $\%$ & $\mathbf{N}$ & $\%$ \\
\hline Faz uso de analgésicos? (antes da auriculoterapia) & 5 & 41,67 & 7 & 58,33 \\
\hline Faz uso de analgésicos? (após auriculoterapia) & 2 & 16,67 & 10 & 83,33 \\
\hline $\begin{array}{l}\text { Após a auriculoterapia, houve diminuição do uso de } \\
\text { analgésicos? }\end{array}$ & 4 & 33,33 & 8 & 66,67 \\
\hline $\begin{array}{l}\text { Após a auriculoterapia, houve redução na dosagem dos } \\
\text { analgésicos? }\end{array}$ & 6 & 50,00 & 6 & 50,00 \\
\hline $\begin{array}{l}\text { Após a auriculoterapia, houve redução na frequência do } \\
\text { uso de analgésicos? }\end{array}$ & 4 & 33,33 & 8 & 66,67 \\
\hline Após a auriculoterapia, houve redução da sua dor? & 9 & 75,00 & 3 & 25,00 \\
\hline
\end{tabular}

Legenda: $\mathrm{N}=12$ participantes.

Fonte: Abdoral PRG, et al., 2021.

Quando perguntados sobre o uso de analgésicos antes das sessões de auriculoterapia, 5 participantes $(41,67 \%)$ relataram fazer uso, enquanto $7(58,33 \%)$ negaram. Após as sessões, quando feita a mesma pergunta, 2 participantes (16,67\%) relataram fazer uso, enquanto $10(83,33 \%)$ negaram. Quanto ao uso de analgésicos após as sessões, 4 participantes (33,33\%) relataram diminuição, enquanto $8(66,67 \%)$ não a perceberam. Quando perguntados sobre a dosagem dos analgésicos utilizados, 6 participantes (50\%) relataram diminuição da dosagem, enquanto outros $6(50 \%)$ negaram. Em relação à frequência no uso desses medicamentos, 4 participantes (33,33\%) relataram diminuição, enquanto $8(66,67 \%)$ negaram.

Verificou-se também diferenças na média da escala visual analógica (EVA) dos participantes que faziam uso de analgésico e dos que não faziam uso (Gráfico 1) e diferença após auriculoterapia sobre o uso da medicação (Gráfico 2). 
Gráfico 1 - Média em porcentagem da EVA antes e depois da sessão de auriculoterapia dos pacientes que usavam e dos que não usavam analgésicos.

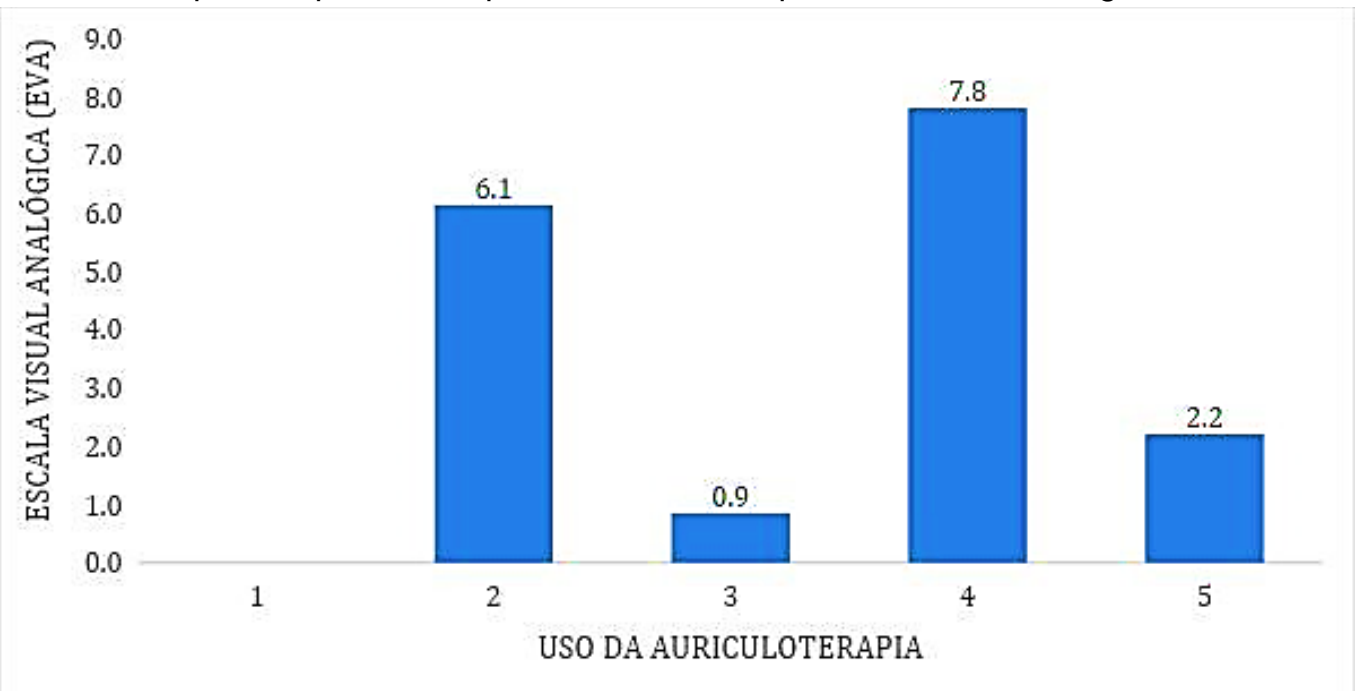

Legenda: $\mathrm{N}=12$ participantes.

Fonte: Abdoral PRG, et al., 2021.

Ao verificar a EVA desses participantes houve redução da intensidade da dor, de moderada para leve, sendo este dado observado no grupo de pacientes que faziam uso de analgésico e do grupo que não fazia uso de analgésico.

Gráfico 2 - Porcentagem do número de pacientes em uso de analgésicos, antes e após as sessões de auriculoterapia.

6

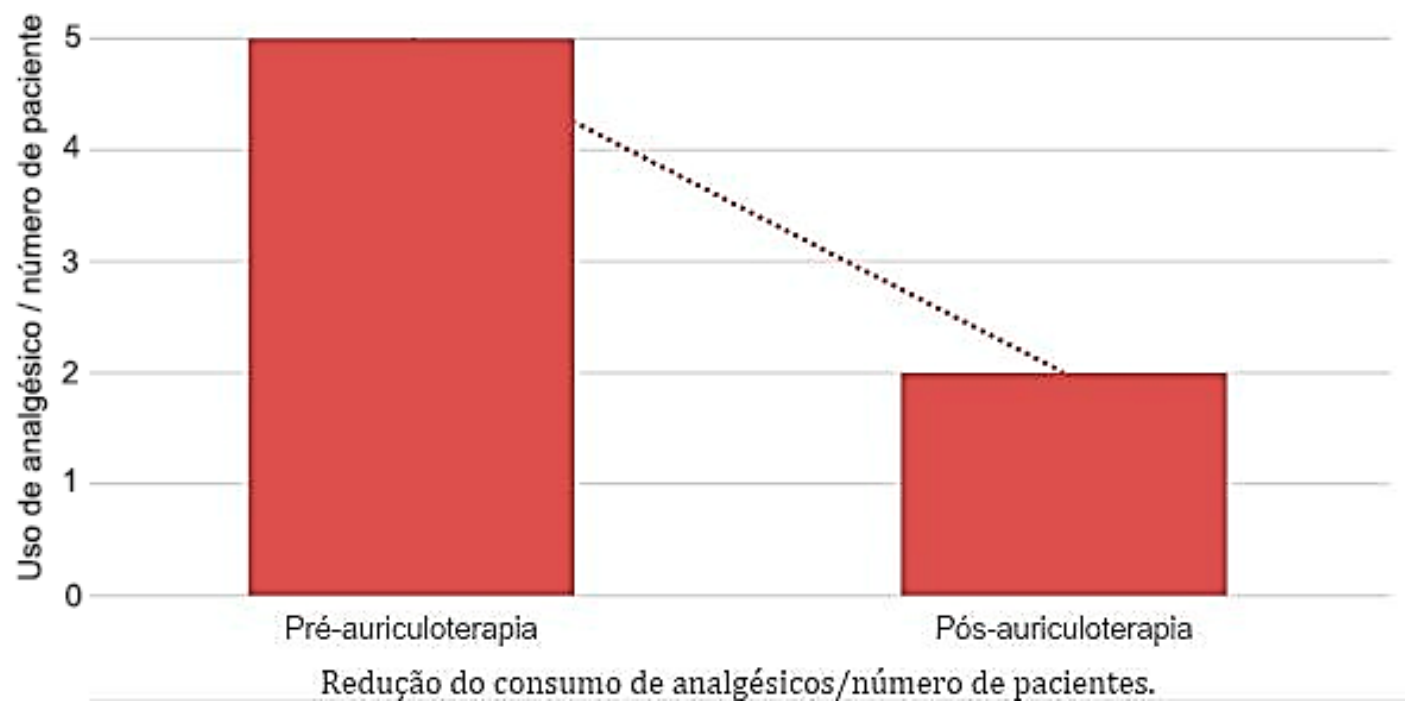

Legenda: $\mathrm{N}=12$ participantes.

Redução do consumo de analgésicos/número de pacientes.

Fonte: Abdoral PRG, et al., 2021.

Quando perguntados sobre o uso de analgésicos antes das sessões de auriculoterapia, 5 participantes $(41,67 \%)$ relataram fazer uso, e ao final da avaliação apenas $2(16,67 \%)$ destes ainda utilizavam. Dado isso, houve uma importante diminuição estatisticamente significante em relação a variável dor antes e após auriculoterapia, como mostrado abaixo (Tabela 3). 
Tabela 3 - Análise das médias de valores obtidos na escala de dor antes e após as sessões de auriculoterapia com Teste T pareado.

\begin{tabular}{ccccc}
\hline Variável & Média & Desvio padrão & Diferença & p valor $^{\star}$ \\
\hline EVA pré & 6,8 & 6,8 & & \\
EVA pós & 1,4 & 1,7 & $-5,4$ & 0,0000 \\
\hline
\end{tabular}

Fonte: Abdoral PRG, et al., 2021.

Quanto à análise da variável EVA antes e após as sessões, observou-se que a dor passou de moderada (média de 6,8 $\pm 6,8$ ) para leve (média de 1,4 $\pm 1,7$ ) após as sessões de auriculoterapia. Ao analisar a diferença entre as médias (Teste T pareado), obteve-se diferença estatisticamente significativa, com $p=0,000$.

\section{DISCUSSÃO}

No presente estudo, a auriculoterapia proporcionou redução estatisticamente significativa na intensidade da dor de acordo com a ferramenta de estudo EVA, ocasionando também na diminuição do uso analgésico, fazendo dessa terapia uma alternativa para o manejo clínico oncológico.

Dados mostrados no estudo, indicam que a maioria dos participantes relataram satisfação com uso da auriculoterapia e que o uso da mesma reduziu consideravelmente sua dor, respectivamente $83,3 \%$ e $75 \%$. Percebeu-se também melhoras em sintomatologias associadas ao tratamento, como a insônia, constipação e ansiedade, o que demonstra importância dessa terapia na melhora da qualidade de vida desses pacientes.

Observou-se que a maioria dos participantes desse estudo era do sexo feminino $(66,7 \%)$, sendo a média de idade 62,3 anos. O diagnóstico prévio mais prevalente foi de câncer de mama (58,3\%) o que está em conformidade com os dados mundiais em que este tipo de neoplasia maligna é mais incidente em mulheres (BRAY F, et al., 2018).

Em pacientes que estão passando por tratamento oncológico, os sintomas dolorosos possivelmente estão associados a ação das condutas farmacológicas, mas também podemos relacionar ao processo de invasão celular da neoplasia, comorbidades e principalmente a fatores psicossomáticos. Estudos vem apontando a dor como o efeito colateral de maior incidência em pacientes oncológicos, sintomas como fadiga e cansaço podem estar associados, podendo afetar diretamente o psicológico do indivíduo o levando a quadros depressivos. Outros sintomas como náusea, vômito e astenia também são relatados. (CAETANO AFP, et al., 2019).

Vale ressaltar, que a maioria relatou a intensidade de dor moderada na avaliação inicial. Sendo que $58,33 \%$ destes referiram não fazer uso de analgésico para o controle da dor, o que demonstra que o manejo álgico desses pacientes ainda é insuficiente. Os profissionais da saúde possuem uma grande dificuldade em classificar os quadros álgicos apresentados pelos pacientes com câncer e ao manejo da dor vivenciada. A modulação dos quadros dolorosos é uma questão de que intriga os profissionais, tal fato estimula a categoria a buscar métodos que apresentem resultados satisfatórios tanto na avaliação quanto no tratamento, proporcionando assim um melhore conforto e qualidade de vida para os pacientes oncológicos (MEIER LK, et al., 2018).

Nesse contexto, destaca-se o uso de terapias complementares, que surgiram como forma de suprir as necessidades dos pacientes, afim de amenizar seus desconfortos e queixas de álgica. Sendo assim, a auriculoterapia, uma vertente da acupuntura, parece ser uma boa proposta terapêutica, visto que em um estudo mostrou-se eficaz na diminuição da dor em pacientes com dor crônica (ZHAO H, et al., 2015). O paciente que está passando por tratamento quimioterápico já está submetido a uma grande carga de medicamentos, fazer uma intervenção não medicamentos e de grande valia para estes indivíduos.

Frente a essa afirmativa, um estudo apontou através da aplicação da auriculoterapia com agulhas na melhora da qualidade vida de pacientes oncológicos, a mesma mostrou-se eficaz na melhora da dor, o que corrobora com resultados encontrados em nossos estudos relacionado a diminuição álgica, sendo nesse 0 uso de sementes (VALLIM ETA, et al., 2019). 
Em um estudo de revisão sistemática e metanálise, demonstrou-se a efetividade da auriculoterapia na dor crônica, dando base de que essa terapêutica é eficaz no que tange o manejo da dor, apoiando para esse estudo que tal técnica também pode ser utilizada no controle da dor oncológica (MOURA CC, et al., 2019).

Importante frisar também a diferença inicial e final da EVA nos participantes, saindo de moderada $(6,8)$ para leve $(1,4)$, o que demonstra uma significante redução da intensidade da dor, resultados esses que comprovam com os encontrados por Kurebayashi LFS (2017), diferenciando-se apenas quanto ao uso de analgésico e a idade média dos participantes que no estudo foi de 35,7 anos e no nosso foi de 62,3 anos.

Desse modo, o estudo também demonstrou que dos 5 participantes que relataram fazer uso do analgésico inicialmente, ao final 2 apenas referiram fazer ainda o uso do mesmo, o que constata uma importante redução na utilização desse tipo de medicação, similarmente aos nossos resultados, um ensaio clinico randomizado com 23 participantes com câncer, que fizeram uso da auriculoterapia para dor, constatou-se não somente a diminuição da intensidade álgica, mas também do consumo de analgésico (RUELA LO, et al., 2018).

A Oncologia Integrava e uma intervenção relativamente nova em nosso país, através dela ocorre uma simbiose do cuidado tradicional e das práticas integrativas e complementares, tais práticas são realizadas respeitando os princípios da bioética, buscando minimizar possíveis danos e deixando como prioridade a autonomia de cada paciente, procurando busca meios para melhorar o seu bem-estar físico e mental (CHAGAS N, et al., 2020).

As terapias integrativas não buscam tratar o paciente de forma isolada, as bases presentes na medicina tradicional chinesa olham o a saúde de forma integram e suas intervenções buscam levar a cura não somente para o corpo mais também para o emocional dos pacientes, seja esse processo de cura levado em decorrência das técnicas aplicadas agirem no corpo de forma sistema, seja pela cuidado individualizado e atencioso presente nos terapeutas integrativos que acabam sendo menos tecnicistas que os demais profissionais da saúde. Tais fatores afetam diretamente a aceitação do paciente sobre a realização da auriculoterapia potencializando o seu resultado (WU X, et al., 2017).

Entretanto, a baixa evidência com o uso da auriculoterapia no manejo da dor oncológica ainda é limitante frente aos resultados inconclusivos sobre a temática, e isso se dá devido aos diferentes tipos de metodologias utilizadas nos estudos, que também foi relatada por outros autores. O processo de desenvolvimento desse estudo apontou alguns fatores limitantes para a conclusão do mesmo, o número pequeno de participantes, ausência de dados importantes relacionadas aos ciclos de quimioterapia na evolução clínica, o que dificultou revelar o tempo de tratamento, óbitos no decorrer da coleta de dados ocasionando perda amostral, entretanto os números de informações coletadas contribuíram de forma substancial no papel da auriculoterapia no manejo da dor e redução do consumo de analgésicos, apesar da nossa amostra ser heterogenia no que concerne aos diferentes tipos de câncer (YEH CH, et al., 2015).

\section{CONCLUSÃO}

A auriculoterapia é uma terapia complementar em saúde, promissora para o manejo da dor em pacientes oncológicos, visto que, houve diminuição no escore de intensidade da dor e redução do consumo de analgésico por parte dos participantes. Logo, é uma técnica que pode ser utilizada pelos profissionais da saúde no âmbito da assistência, com intuito de expandir a prática terapêutica, a fim de melhor atender o paciente. A necessidade na continuidade de outras pesquisas do assunto aqui tratado ficou muito presente ao analisar esse estudo.

\section{REFERÊNCIAS}

1. ARTIOLI DP, et al. Auriculoterapia: neurofisiologia, pontos de escolha, indicações e resultados em condições dolorosas musculoesqueléticas: revisão sistemática de revisões. Sociedade Brasileira para o Estudo da Dor, 2019; 2: 356-361.

2. MINISTÉRIO DA SAÚDE. Práticas Integrativas e Complementares (PICS): quais são e para que servem, 2021. Disponível em antigo.saude.gov.br/saude-de-a-z/praticas-integrativas-e-complementares. Acessado em: 03 de agosto de 2021. 
3. MINISTÉRIO DA SAÚDE. Estimativa 2020: incidência de câncer no Brasil, Instituto Nacional de Câncer José Alencar Gomes da Silva. - Rio de Janeiro: INCA, 2019. Disponível em: www.inca.gov.br/publicacoes/livros/estimativa-2020incidencia-de-cancer-no-brasil. Acessado em: 03 de agosto de 2021.

4. BRAY F, et al. Global cancer statistics 2018:GLOBOCAN estimates of incidence and mortality worldwide for 36 cancers in 185 countries. CA: a cancer journal for clinicians, 2018; 68: 394-424.

5. CAETANO AFP, et al., Associação entre Prática de Atividade Física, Dor e Fadiga nos Pacientes em Tratamento Quimioterápico. O Mundo da Saúde, 2020; 1: 35-44.

6. CHAGAS N, et al. Acendendo as Luzes: uma inovação no Cuidado a Saúde dos Pacientes Oncológicos, Familiares e Equipe. Saúde em Redes2020; 6: 155-162.

7. Garcia TR, et al. Classificação Internacional para a Prática de Enfermagem - CIPE Versão 2017. Porto Alegre: Artmed, 2018.

8. KIPEL AG, et al. Práticas de enfermagem no manuseio da dor em hospitais de um município de Santa Catarina. Rev Dor. 2015.

9. KUREBAYASHI LFS, et al. Auriculoterapia para redução de ansiedade e dor em profissionais de enfermagem: ensaio clínico randomizado. Revista Latino-Americana de Enfermagem, 2017; 25.

10. LI T, et al. Clinical efficacy and safety of acupressure on low back pain: A systematic review and meta-analysis. Evidence-Based Complementary and Alternative Medicine, 2021;21.

11. LUIZ, MM et al. Cuidados paliativos em enfermagem ao idoso em UTI: uma revisão integrativa/Palliative nursing care in the elderly in UCI: an integrative review. Revista de Pesquisa Cuidado é Fundamental Online, 2018; 2: 585-592.

12. MATOS, LC et al. Understanding Traditional Chinese Medicine Therapeutics: An Overview of the Basics and Clinical Applications. In: Healthcare. Multidisciplinary Digital Publishing Institute, 2021.

13. MEIER LK, et al. Manejo da dor de pacientes oncológicos residentes em área de estratégia de saúde da família. Revista Interdisciplinar de Promoção da Saúde, 2018; 3: 163-168.

14. MOURA CDC. et al. Auricular acupuncture for chronic back pain in adults: a systematic review and metanalysis. Rev Esc Enferm USP. 2019.

15. MOURA, CC et al. Acupuntura auricular para dor crônica nas costas em adultos: revisão sistemática e metanálise. Revista da Escola de Enfermagem da USP, 2019; 53.

16. PENG, $L$ et al. Transauricular vagus nerve stimulation at auricular acupoints Kindey (CO10), Yidan (CO11), Liver (CO12) and Shenmen (TF4) can induce auditory and limbic cortices activation measured by fMRI. Hearing research, 2018; 359: 1-12.

17. PEREIRA FEM, MOREIRA DVQ. A Influência da Acupuntura e Auriculoterapia nos Biomarcadores Ureia e CreatininaUm Estudo de Caso. Rev Bras Terap e Saúde, 2017; 8: 15-19.

18. Queiroga HM, et al. Avaliação de náusea e êmese em pacientes sob quimioterapia em uma Unidade de Alta Complexidade Saúde de Vitória da Conquista/BA. Rev. Bras. Pesq. Saúde [Internet], 2017.

19. RODRIGUES CL, GUSMAN, GS. Uso De Terapias Alternativas E Complementares Por Pacientes Oncológicos Em Quimioterapia. Revista Científica Univiçosa, Minas Gerais, 2018; 10: 315-320.

20. RUELA LO, et al. Effectiveness of auricular acupuncture in the treatment of cancer pain: randomized clinical trial. RevEsc Enferm USP. 2018.

21. SANTOS MO. Estimativa 2018: incidência de câncer no Brasil. Revista Brasileira de Cancerologia, 2018; 64: 119-120.

22. SAYED D, et al. Retrospective analysis of intrathecal drug delivery: outcomes, efficacy, and risk for cancer-related pain at a high volume Academic Medical Center. Neuromodulation: Technology at the Neural Interface, 2018; 21: 660-664.

23. VALLIM ETA, et al. Auriculotherapy with Needles to Improve the Quality of Life of Cancer Patients: An Integrative Literature Review. Rev Fund Care Online.2019.

24. WU X, et al. Effectiveness of acupuncture and related therapies for palliative care of cancer: overview of systematic reviews. Deutsche Zeitschrift für Akupunktur, 2017; 2: 28-29.

25. YEH CH, et al. Pilot Randomized Controlled Tril of Auricular Point Acupressure to Manage Symptom Clusters of Pain, Fatigue, and Disturbed Sleep in Breast Cancer Patients, Cancer Nursing TM, Pittsburgh, 2015; 1: 1-9.

26. ZHAO H, et al. Auricular therapy for chronic pain management in adults: A synthesis of evidence: Complementary Therapies in Clinical Practice, China, 2015; 21: 68-78. 